\title{
The efficacy of levomilnacipran ER across symptoms of major depressive disorder: a post hoc analysis of 5 randomized, double-blind, placebo-controlled trials
}

\author{
Roger S. McIntyre, ${ }^{1,2 *}$ Carl Gommoll, ${ }^{3}$ Changzheng Chen, ${ }^{4}$ and Adam Ruth ${ }^{5}$
}

\footnotetext{
${ }^{1}$ Department of Psychiatry and Pharmacology, University of Toronto, Toronto, Ontario, Canada

${ }^{2}$ Mood Disorders Psychopharmacology Unit, University Health Network, Toronto, Ontario, Canada

${ }^{3}$ Department of Clinical Development, Forest Research Institute, Inc. (an Allergan affiliate), Jersey City, New Jersey, USA

${ }^{4}$ Department of Statistical Science, Forest Research Institute, Inc. (an Allergan affiliate), Jersey City, New Jersey, USA

${ }^{5}$ Prescott Medical Communications Group, Chicago, Illinois, USA
}

Objective. A post hoc analysis evaluated the effects of levomilnacipran ER on individual symptoms and symptom domains in adults with major depressive disorder (MDD).

Methods. Data were pooled from 5 Phase III trials comprising 2598 patients. Effects on depression symptoms were analyzed based on change from baseline in individual Montgomery-Åsberg Depression Rating Scale (MADRS) item scores. A1dditional evaluations included resolution of individual symptoms (defined as a MADRS item score $\leq 1$ at end of treatment) and concurrent resolution of all 10 MADRS items, all MADRS ${ }_{6}$ subscale items, and all items included in different symptom clusters (Dysphoria, Retardation, Vegetative Symptoms, Anhedonia).

Results. Significantly greater mean improvements were found on all MADRS items except Reduced Appetite with levomilnacipran ER treatment compared with placebo. Resolution of individual symptoms occurred more frequently with levomilnacipran ER than placebo for each MADRS item (all $P<.05$ ), with odds ratios (ORs) ranging from 1.26 to 1.75; resolution of all 10 items was also greater with levomilnacipran $\mathrm{ER}(\mathrm{OR}=1.57 ; P=.0051)$. Significant results were found for the $\mathrm{MADRS}_{6}$ subscale $(\mathrm{OR}=1.73 ; P<.0001)$ and each symptom cluster (OR range, 1.39 [Vegetative Symptoms] to 1.84 [Retardation]; all clusters, $P<.01$ ).

Conclusion. Adult MDD patients treated with levomilnacipran ER improved across a range of depression symptoms and symptom domains.

Received 1 June 2015; Accepted 27 August 2015; First published online 13 June 2016

Key words: Antidepressant, depression, remission, symptom clusters, treatment.

\section{Introduction}

Although a diagnosis of major depressive disorder (MDD) requires the presence of several key symptoms, there is

* Address for correspondence: Roger S McIntyre, MD, FRCPC, Mood Disorders Psychopharmacology Unit, University Health Network,

399 Bathurst Street, Toronto, ON M5T 2S8, Canada.

(Email: roger.mcintyre@uhn.ca)

This analysis and the studies included in the analysis were supported by Forest Laboratories, LLC, an Allergan affiliate.

The authors thank William Greenberg for his contributions to the planning and early development of this manuscript. At the time of the studies, Dr. Greenberg was a full-time employee of Forest Research Institute, an Allergan affiliate. Writing and editorial assistance was provided by Mildred Bahn at Prescott Medical Communications Group (Chicago, IL), a contractor for Forest Research Institute. wide variation among individual patients in terms of clinical presentation. ${ }^{1}$ The symptomatic heterogeneity of MDD was recently documented in a post hoc analysis of the Sequenced Treatment Alternatives to Relieve Depression $\left(\mathrm{STAR}^{*} \mathrm{D}\right)$ trial, which identified 1030 unique symptom profiles based on responses from 3703 patients on the 16-item Quick Inventory of Depression Symptoms (QIDS-16) scale. ${ }^{2}$ Given such findings, it is not surprising that individuals respond differently to available antidepressants, and optimization of treatment may require selecting medications that target specific types of symptoms. ${ }^{3}$ One such approach has been to identify groups of related symptoms and subsequently evaluate the effects of various pharmacotherapies on the pre-specified symptom clusters. ${ }^{4-7}$ Although different instruments and statistical 
models have been used in this area of research, studies have generally indicated that symptom clusters may be an important index of treatment response.

Failure to achieve full symptom remission is also a major challenge in MDD. Residual symptoms after acute treatment have been associated with more recurrent episodes, greater functional impairment and disability, and increased economic costs. ${ }^{8}$ Improvements in different symptom domains may have varying effects on social and occupational functioning, as suggested by results indicating that remission of select mood and anxiety symptoms (in the absence of pain) had a stronger association with functional improvements than remission of insomnia symptoms or the absence of pain alone. ${ }^{9}$ Residual fatigue ${ }^{10}$ and cognitive problems ${ }^{11}$ have also been associated with greater functional impairment, providing the impetus for characterizing and specifically targeting symptom domains in MDD.

Levomilnacipran extended-release (ER) is a serotonin and norepinephrine reuptake inhibitor (SNRI) that is currently approved for the treatment of MDD in adults. In 3 pivotal trials ${ }^{12-14}$ and 1 proof-of-concept trial, ${ }^{15}$ the superiority of levomilnacipran ER to placebo was demonstrated on the basis of statistically significant reductions in overall depression symptomatology, as assessed by mean change from baseline in the Montgomery-Åsberg Depression Rating Scale (MADRS) ${ }^{16}$ total score. The current post hoc analysis of 5 studies, ${ }^{12-15,17}$ all of which used MADRS total score as the primary efficacy measure, was conducted in order to evaluate the effects of levomilnacipran ER on individual symptoms and on different symptom clusters. Preliminary analyses were also conducted to explore the relationship between symptom remission and improvements in functional impairment.

\section{Methods}

\section{Study designs}

Post hoc analyses were conducted on data pooled from 5 randomized, double-blind, placebo-controlled trials of levomilnacipran ER. These included 2 fixed-dose $\mathrm{f}^{12,13}$ and 3 flexible-dose trials ${ }^{14,15,17}$ in which patients received 8 weeks ${ }^{12-14,17}$ or 10 weeks ${ }^{15}$ of double-blind treatment with levomilnacipran ER (40-120 mg/day) or placebo.

Methods for all 5 trials have been previously published. Briefly, they included adults with MDD who had a current major depressive episode. Patients entering the 4 U.S. studies were required to have a MADRS total score $\geq 26^{13}$ or $\geq 30,{ }^{12,14,17}$ with 1 study also requiring a Clinical Global Impression of Severity score $\geq 4$. ${ }^{13}$ Patients in the non-U.S. study $^{15}$ were required to have a 17-item Hamilton Rating Scale for Depression $\left(\mathrm{HAMD}_{17}\right)$ total score $>22$ and Sheehan Disability Scale (SDS) total score $\geq 10$ with at least 1 subscale score $\geq 6$. Patients with a principal Diagnostic and Statistical Manual of Mental Disorders, Fourth Edition, Text Revision (DSM-IV-TR)
Axis I diagnosis other than MDD, social anxiety disorder, generalized anxiety disorder, or specific phobia were excluded from the U.S. studies. The non-U.S. study also excluded patients if the onset of an allowed comorbid psychiatric illness (eg, panic disorder, obsessive compulsive disorder, generalized anxiety disorder, posttraumatic stress disorder) preceded the current depressive episode. All of the studies excluded patients who had a history of nonresponse to $\geq 2$ antidepressants after adequate treatment or a significant risk of suicide based on investigator judgment or formal assessment (eg, ColumbiaSuicide Severity Rating Scale, Mini-International Neuropsychiatric Interview).

\section{Post hoc analyses}

Analyses were conducted in the pooled ITT Population, which was defined as all randomized patients who received $\geq 1$ dose of double-blind study medication and had $\geq 1$ post-baseline MADRS assessment. For each MADRS item, the least squares mean change from baseline at end of treatment and at study visits (Weeks $1,2,4,6$, and 8) were analyzed using an analysis of covariance (ANCOVA) model with study, pooled study sites, and treatment as factors and baseline item scores as covariates; missing data were imputed using the last observation carried forward approach. Treatment effect sizes were estimated using the Cohen's $d$ calculation.

Symptom resolution, defined as no/minimal symptoms after treatment (MADRS item score $\leq 1$ ), was analyzed in patients from the ITT Population who completed the final study visit at Week 8 (Week 10 in the non-U.S. study). Symptom resolution was analyzed for each individual MADRS item in all study completers, as well as in completers who had moderate-to-severe symptom severity at baseline, defined as a MADRS item score $\geq 4$. These cut-offs were based on the defined scale steps (ie, scores 0, 2, 4, and 6) for the MADRS items; for each MADRS item, these steps are accompanied by descriptors that suggest no/minimal symptoms (score 0 or 1 ), mild symptoms (score 2 or 3 ), moderate symptoms (score 4 or 5 ), or severe symptoms (score 6). Also analyzed was the percentage of patients after treatment who had no/minimal symptoms on all 10 MADRS items and on all 6 items that constitute the MADRS ${ }_{6}$ Subscale (items 1 [Apparent Sadness], 2 [Reported Sadness], 3 [Inner Tension], 7 [Lassitude], 8 [Inability to Feel], and 9 [Pessimistic Thoughts]). ${ }^{18}$

Based on symptom clusters that have been previously defined in the literature, ${ }^{4}$ analyses were conducted to determine the percentage of patients with remission on all items in each of the following clusters: Dysphoria (items 2 [Reported Sadness], 9 [Pessimistic Thoughts], 10 [Suicidal Thoughts]); Retardation (items 1 [Apparent Sadness], 6 [Concentration Difficulties], 7 [Lassitude], 
8 [Inability to Feel]); and Vegetative Symptoms (items 3 [Inner Tension], 4 [Reduced Sleep], 5 [Reduced Appetite]). Since anhedonia is a core feature of MDD, ${ }^{19}$ an Anhedonia cluster (items 5 [Reduced Appetite], 7 [Lassitude], 8 [Inability to Feel], 10 [Suicidal Thoughts]) was defined for this post hoc analysis. For all symptom resolution outcomes, odds ratios (ORs) with $95 \%$ confidence intervals $(95 \% \mathrm{CI})$ were estimated using

\begin{tabular}{|lcc|}
\hline \multicolumn{2}{|c|}{ TABLE 1. Patient demographics and baseline characteristics } \\
\hline Characteristic & $\begin{array}{l}\text { Placebo } \\
\mathrm{n}=1032\end{array}$ & $\begin{array}{c}\text { Levomilnacipran ER } \\
\mathrm{n}=1566\end{array}$ \\
& & \\
\hline Age, years, mean (SD) & $43.5(12.7)$ & $42.7(12.9)$ \\
Women, $\mathrm{n}(\%)$ & $660(64.0)$ & $997(63.7)$ \\
White, $\mathrm{n}$ (\%) & $846(82.1)$ & $1228(78.4)$ \\
BMI, $\mathrm{kg} / \mathrm{m}^{2}$, mean (SD) & $28.2(5.7)$ & $28.1(5.5)$ \\
MADRS scores, mean (SD) & & \\
Total & $33.3(4.6)$ & $33.8(4.5)$ \\
Item 1: Apparent Sadness & $3.9(0.8)$ & $4.0(0.8)$ \\
Item 2: Reported Sadness & $4.1(0.7)$ & $4.1(0.7)$ \\
Item 3: Inner Tension & $3.4(1.0)$ & $3.4(0.9)$ \\
Item 4: Reduced Sleep & $3.8(1.1)$ & $3.8(1.1)$ \\
Item 5: Reduced Appetite & $2.4(1.7)$ & $2.5(1.7)$ \\
Item 6: Concentration & $3.8(0.9)$ & $3.8(0.9)$ \\
Difficulties & & \\
Item 7: Lassitude & $3.8(0.8)$ & $3.8(0.8)$ \\
Item 8: Inability to Feel & $3.9(0.8)$ & $4.0(0.8)$ \\
Item 9: Pessimistic Thoughts & $3.2(1.1)$ & $3.3(1.1)$ \\
Item 10: Suicidal Thoughts & $1.0(1.1)$ & $1.1(1.2)$ \\
\hline
\end{tabular}

BMI, body mass index; ER, extended release; MADRS, Montgomery-Åsberg Depression Rating Scale; SD, standard deviation. a logistic regression model with treatment as a factor and baseline scores as covariates.

\section{Results}

In the pooled ITT Population, demographics and baseline characteristics were similar between treatment groups (Table 1). The highest mean baseline scores were found for MADRS items 1 (Apparent Sadness), 2 (Reported Sadness), and 8 (Inability to Feel).

In this overall population, significantly greater mean improvements from baseline to end of treatment were seen with levomilnacipran ER compared with placebo on all MADRS items except Reduced Appetite (Figure 1). The largest effect sizes were found in Apparent Sadness, Reported Sadness, Concentration Difficulties, and Inability to Feel (all Cohen's $d \geq 0.2$ ). By Week 1 of double-blind treatment, statistically significant differences between treatment groups were detected for Apparent Sadness, Reported Sadness, Lassitude, and Inability to Feel (Figure 2). By Week 2, significant differences in favor of levomilnacipran ER were detected for Concentration Difficulties, Pessimistic Thoughts, and Suicidal Thoughts.

Among study completers, the percentage of patients with symptom resolution (ie, no/minimal symptoms, defined as MADRS item score $\leq 1$ ) at end of treatment was significantly higher with levomilnacipran ER than placebo for each MADRS item, with ORs ranging from 1.26 (Reduced Sleep, Reduced Appetite) to 1.75 (Apparent Sadness) (Figure 3A). In study completers who had moderate-to-severe depression symptoms at baseline

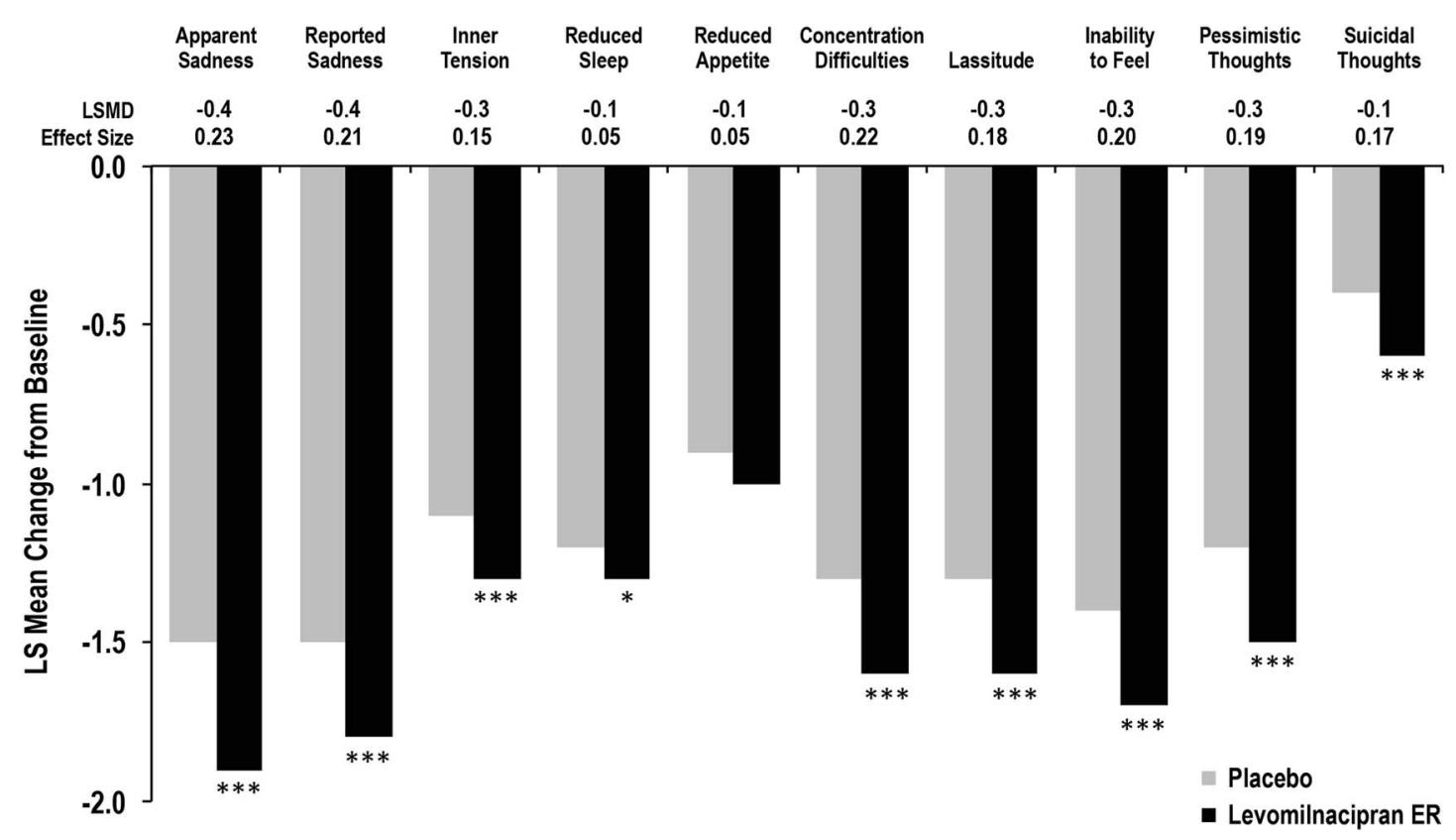

FIGURE 1. Mean improvements in MADRS items at end of treatment. ${ }^{*} P<.05 ;{ }^{* *} P<.001$ versus placebo. ER, extended release; LS, least squares; LSMD, least squares mean difference between treatment groups; MADRS, Montgomery-Åsberg Depression Rating Scale. 


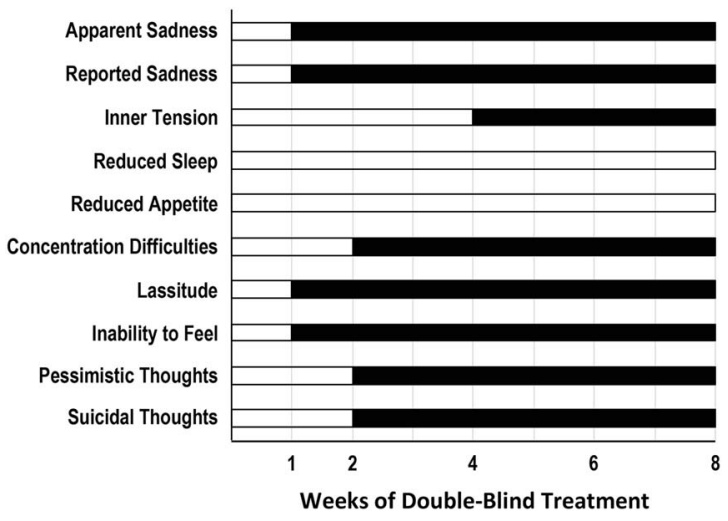

FIGURE 2. Statistical separation between levomilnacipran ER and placebo in MADRS items by study visit. Analysis was based on least squares mean changes from baseline in individual MADRS item scores using last observation carried forward. Color change from white to black indicates the first study visit at which a statistically significant $(P<.05)$ difference between treatment groups was detected and remained significant at every subsequent study visit. Analysis does not include Week 10 data from the non-U.S. study. ${ }^{15}$ ER, extended release; MADRS, Montgomery-Åsberg Depression Rating Scale.

(MADRS item score $\geq 4$ ), the percentage of patients with symptom resolution was significantly higher with levomilnacipran ER versus placebo on all MADRS items except for Suicidal Thoughts, which only included 85 patients (Figure 3B). The ORs in the group with moderate-to-severe baseline symptoms were similar to those found in the overall completer population, ranging from 1.32 (Reduced Sleep) to 2.07 (Suicidal Thoughts).

The percentage of patients with concurrent resolution

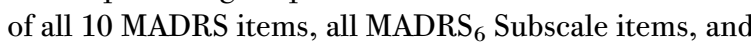
all items in each symptom cluster (Dysphoria, Retardation, Vegetative Symptoms, Anhedonia) was also significantly greater with levomilnacipran ER compared with placebo (Figure 4). The largest between-group rate differences were found in the Retardation cluster $(\mathbf{8 . 3 \%}$; $\mathrm{OR}=1.84 ; P<.0001)$ and Anhedonia cluster $(8.3 \%$; $\mathrm{OR}=1.65 ; P<.0001)$.

\section{Discussion}

A previous post hoc analysis of the 5 clinical trials included in this report showed that adults with MDD who were treated with levomilnacipran ER had clinically relevant and statistically significant improvements in overall depression severity as assessed using MADRS total score, including greater rates of response and remission. ${ }^{20}$ The current post hoc analysis was conducted using individual MADRS items as outcome measures in order to assess the effects of levomilnacipran ER on specific symptoms and the probability of achieving resolution of individual symptoms and symptom clusters.

Results of the current analysis showed significantly greater mean improvements on 9 of the 10 MADRS items, with the highest Cohen's effect sizes found in Apparent Sadness, Concentration Difficulties, Reported Sadness, and Inability to Feel. Onset of symptom improvement with levomilnacipran ER was rapid for Apparent Sadness, Reported Sadness, Lassitude, Inability to Feel, Concentration Difficulties, Pessimistic Thoughts, and Suicidal Thoughts as indicated by statistical separation from placebo within the first 2 weeks of double-blind treatment. These early improvements may be clinically relevant, as suggested by an analysis of $\mathrm{STAR}^{*} \mathrm{D}$ data, which found that Week 2 improvements in similar QIDS-16 items (ie, feeling sad, view of myself, energy level, feeling slowed down, feeling restless) were significantly associated with achieving remission of overall depression at Week $14 .^{21}$ The broader importance of early symptom improvement has been established in a meta-analysis of 41 MDD clinical trials that included different classes of antidepressant medications. ${ }^{22}$ Results of this analysis showed that a $\geq 20 \%$ decrease in overall depression severity at Week 2 was highly predictive of "stable" response and symptom remission, as observed at Week 4 and maintained at all subsequent study visits.

Results indicating resolution of each MADRS item (ie, score $\leq 1$ at Week $8 / 10$ of double-blind treatment) suggest a possible relationship between early levomilnacipran ER effects and end-of-treatment outcomes, although this association was not formally investigated in the current post hoc analysis. The 7 MADRS items that had shown significant treatment-placebo differences at Week 1 or 2 were the same items with the greatest ORs for symptom resolution at end of treatment in the overall completer population (range, 1.42 [Lassitude] to 1.75 [Apparent Sadness]). However, the high percentage of patients in this analysis who had no/minimal symptoms for the Suicidal Thoughts item (>85\% in each treatment group), coupled with the relatively low mean score changes for both placebo and levomilnacipran ER (-0.4 and -0.6 , respectively), suggest possible ceiling and floor effects that may have been due to low mean baseline scores, which probably resulted from the exclusion of patients with suicidal ideation or behavior. To adjust for such effects, symptom resolution was analyzed in study completers who had moderate-to-severe symptoms at baseline, defined as MADRS item scores of 4 or higher. Three items (ie, Reduced Appetite, Pessimistic Thoughts, and Suicidal Thoughts) were found for which $<50 \%$ of the completer population had a baseline score $\geq 4$. The ORs for these 3 items in patients with moderate-to-severe baseline symptoms $(1.62,1.67$, and 2.07, respectively) were higher than in the overall completer population $(1.26,1.43$, and 1.69 , respectively), indicating the need for clinicians to inquire about the severity of specific depression-related symptoms when making treatment decisions. 


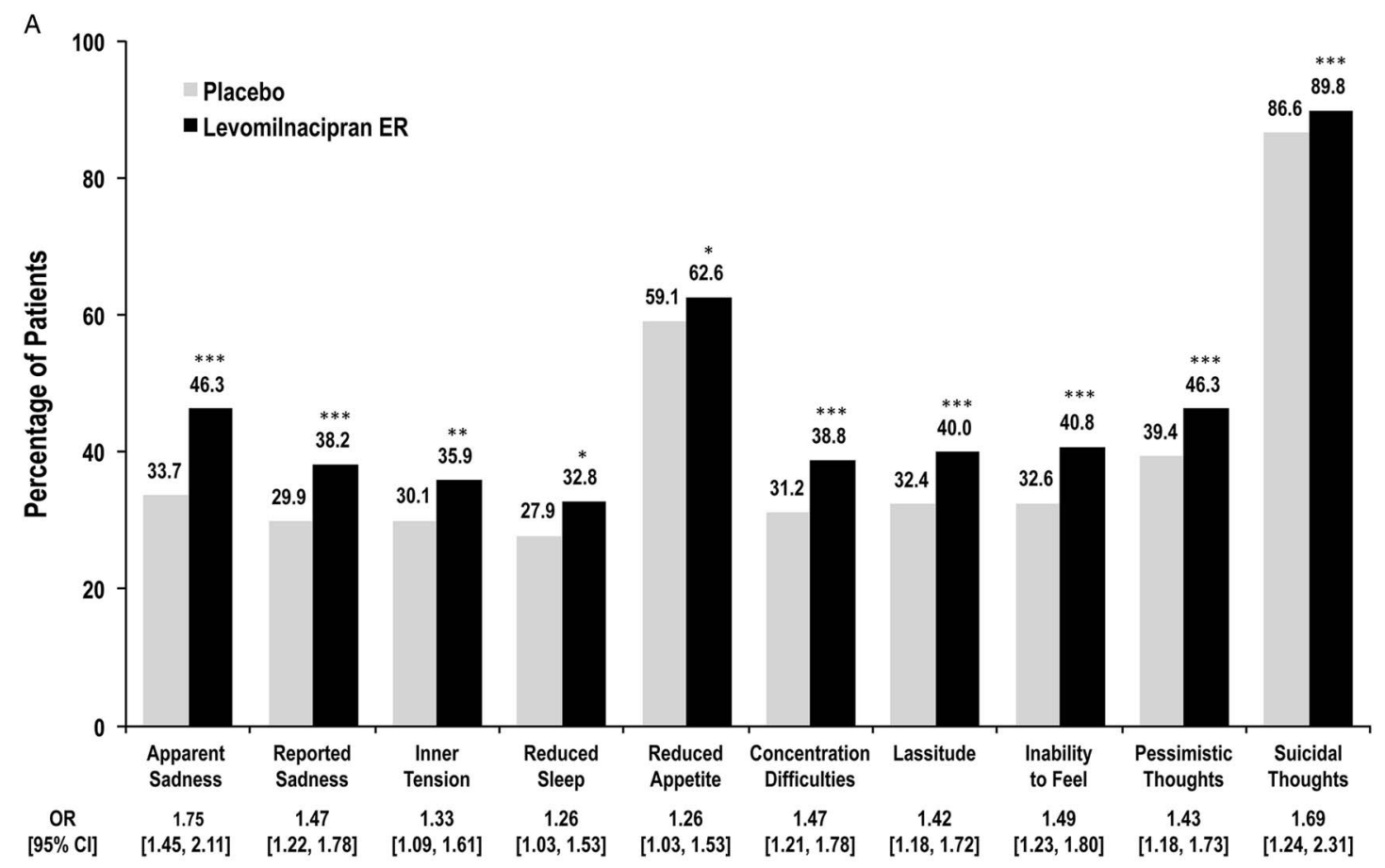

B

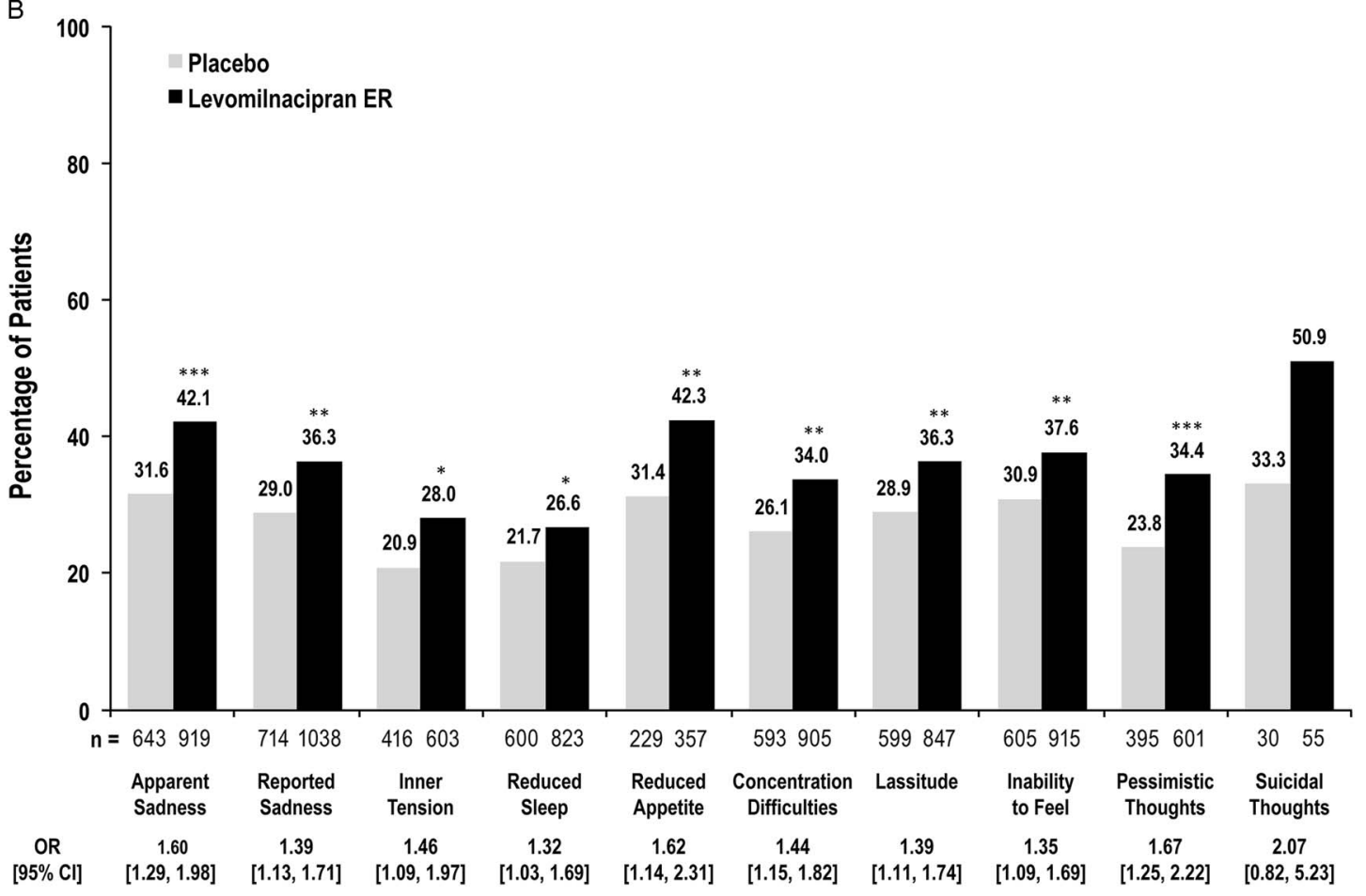

FIGURE 3. Percentage of patients with no/minimal symptoms at end of treatment. No/minimal symptoms defined as a MADRS item score $\leq 1$ at Week $8 / 10$. Analyses were conducted in the following groups: (A) all patients who completed the study (placebo, $n=834$; levomilnacipran ER, $n=1181$ ); and (B) study completers who had moderate-to-severe symptoms at baseline, defined as a MADRS item score $\geq 4$ ( $\mathrm{n}$-values for each item indicated in the figure). ${ }^{*} P<.05$; ${ }^{* *} P<.01 ;{ }^{* *} P<.001$ versus placebo. $\mathrm{Cl}$, confidence interval; ER, extended release; MADRS, Montgomery-Asberg Depression Rating Scale.

Perhaps more important than the analyses based on individual MADRS item scores was the analysis of concurrent symptom resolution on all 10 MADRS items. Although the percentage of patients who met this definition was significantly higher with levomilnacipran ER than with placebo $(11.2 \%$ vs $7.8 \% ; P<.01)$, the rates in both groups were lower than the percentage of patients who had met the more conventional definition of MADRS 


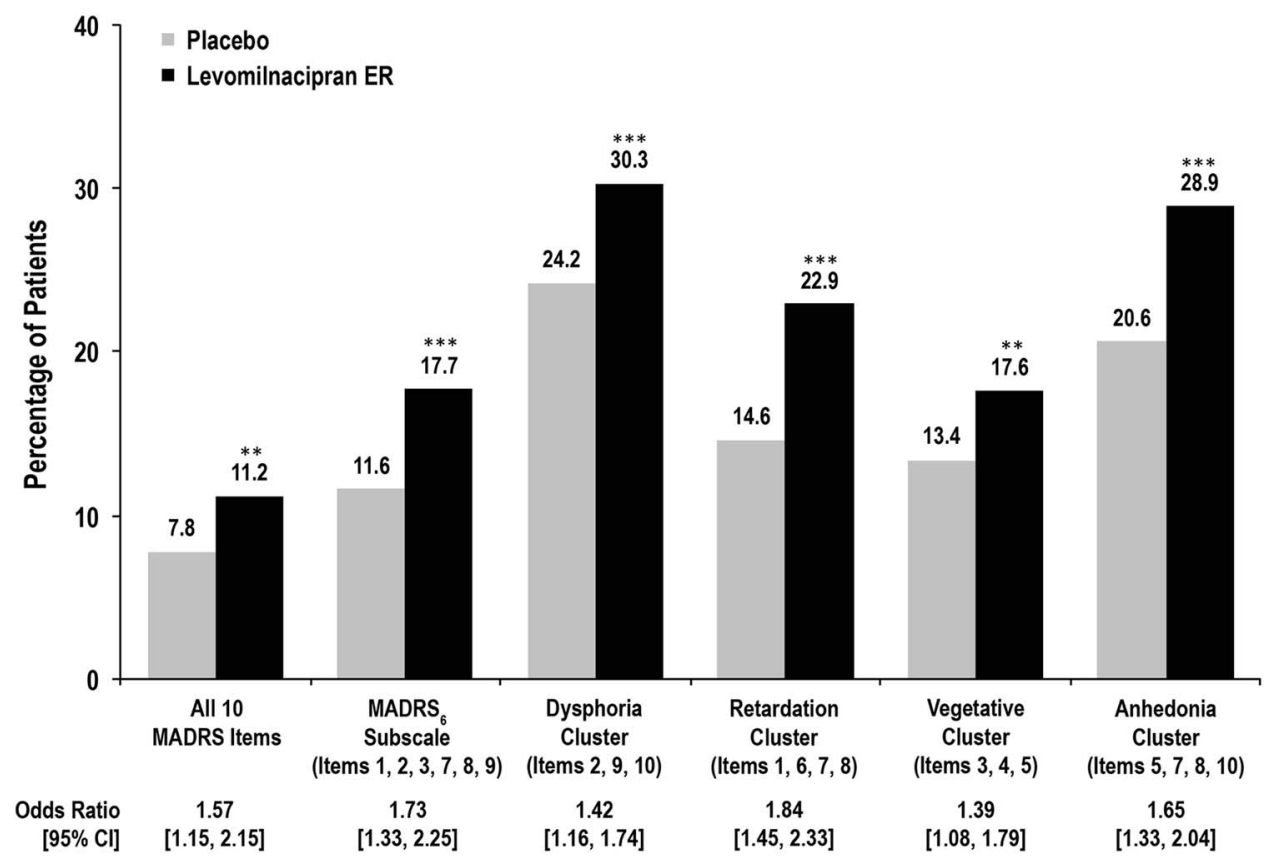

FIGURE 4. Percentage of patients with no/minimal symptoms across different symptom clusters. ${ }^{* *} P<.01 ;{ }^{* *} P<.001$ versus placebo. $\mathrm{Cl}$, confidence interval; ER, extended release; MADRS, Montgomery-Åsberg Depression Rating Scale.

remission (total score $\leq 10)^{23}$ in the previous post hoc analysis $(27.7 \%$ vs $21.5 \% ; P<.05) .{ }^{20}$ This difference suggests that although the criteria for remission may seem mathematically similar between the 2 post hoc analyses, the criteria used in the present analysis represent a more stringent benchmark of complete symptom remission. The difference between MADRS item-level remission and MADRS total score remission also suggests that there may have been a heterogeneity of treatment response in the levomilnacipran ER studies. In other words, results from the current analysis suggest that some patients with a MADRS total score $\leq 10$ may have had complete remission (score of 0 ) on certain clusters of items and residual symptoms (score $\geq 2$ ) on other items, with likely variation across individual patients.

The question of whether specific types or clusters of symptoms may have been more responsive to levomilnacipran ER treatment was addressed by conducting analyses in symptom domains that have been previously reported in the literature. These included the MADRS subscale, ${ }^{18,24} 3$ symptom clusters (Dysphoria, Retardation, Vegetative Symptoms) that were derived using a factor analysis of individual MADRS items, ${ }^{4}$ and an Anhedonia cluster defined for this post hoc analysis to include MADRS items 5, 7, 8, and 10 based on symptoms that indicate lack of pleasure or volition. ${ }^{25}$ Although changes in factor scores have been used to evaluate the effects of antidepressant treatment and nonpharmacologic therapies on the Dysphoria, Retardation, and Vegetative Symptoms clusters, ${ }^{26-28}$ this is the first analysis (to our knowledge) to examine symptom resolution within the clusters. The results indicate that a significantly higher percentage of levomilnacipran ER-treated patients than placebo-treated patients had substantial improvements in all 3 symptom domains, as well as in the Anhedonia cluster and in core depression symptoms as represented by the $\mathrm{MADRS}_{6}$ subscale.

Results from our analyses, however, also suggest that levomilnacipran ER may have had differential effects across the various symptom domains. For example, the largest treatment effect in this post hoc analysis was found in the Retardation cluster $(\mathrm{OR}=1.84)$, which was greater than the effect seen in patients who had resolution on core items comprising the $\mathrm{MADRS}_{6}$ subscale $(\mathrm{OR}=1.73)$ or even on all 10 MADRS items $(\mathrm{OR}=1.57)$. These results may have been partly due to the effects of levomilnacipran ER on symptoms that are associated with deficits in noradrenergic transmission such as reduced energy and alertness, difficulties with concentration and attention, and loss of interest in surroundings and activity. ${ }^{3}$

Conversely, although still statistically significant, the smallest treatment effect was seen in the Vegetative Symptoms cluster $(\mathrm{OR}=1.39)$, which includes items (ie, Inner Tension, Reduced Sleep, Reduced Appetite) that are associated with increased noradrenergic activity and activation of the stress response system. ${ }^{29}$ However, 
some patients-such as those with atypical depressionhave symptoms that are associated with decreased noradrenergic activity, including feelings of disconnectedness and emptiness, lethargy, excessive sleep, increased food intake, and weight gain. Given the effects of levomilnacipran ER on certain items in the Retardation cluster (eg, Concentration Difficulties, Lassitude, Inability to Feel), it seems reasonable to surmise that this agent would have similar effects on depression-related hypersomnolence and overeating. This presumption could not be tested in the current patient population, since such symptom assessments are not included in either of depression scales (MADRS, $\mathrm{HAMD}_{17}$ ) used in the levomilnacipran ER studies. However, the known limited effects of serotonergic antidepressants on atypical depression, ${ }^{30}$ coupled with findings that symptoms of atypical depression significantly predict treatment response to the SNRI duloxetine, ${ }^{31}$ suggest that MDD patients who have increased sleep and/or appetite may benefit from medications that elevate norepinephrine levels.

In addition to symptom improvement, patients who received levomilnacipran ER in the trials included herein had significant improvements in functional impairment, as demonstrated in 2 previously published analyses based on different SDS outcomes including response, remission, and categorical shifts. ${ }^{32,33}$ Such findings are not surprising, since a number of other MDD studies have shown that reduced symptom severity is associated with increased functional ability. ${ }^{34}$ The results of the current post hoc analysis, however, may shed some light on the types of symptoms that may have been driving SDS improvements in the levomilnacipran ER studies. Of the 8 MADRS items that were included in the 2 groups with the largest treatment effects, Concentration Difficulties was unique to the Retardation cluster, and Apparent Sadness, Lassitude, and Inability to Feel were common to both the Retardation cluster and the $\mathrm{MADRS}_{6}$ subscale. Further analyses would be needed to evaluate how improvements in these types of symptoms might affect functional outcomes.

The major limitation of these analyses is that they were conducted post hoc, and no efficacy outcomes based on individual MADRS items had been defined a priori. In addition, any inferences about the differential treatment effects on symptom clusters are limited to acute treatment with levomilnacipran ER; such differences may be more or less apparent with longer treatment durations. Because there were no active comparators in any of the trials, no conclusions can be drawn regarding the effects of levomilnacipran ER relative to other antidepressants. Finally, findings from this study population may not be generalizable to more diverse clinic- and hospital-based populations.

\section{Conclusions}

This post hoc analysis of MADRS item data from 5 Phase III studies indicates that patients treated with levomilnacipran ER versus placebo had significantly greater mean improvements and higher rates of symptom resolution across a range of depression symptoms and in different symptom domains. Moreover, levomilnacipran ERtreated patients had significantly greater odds of achieving resolution across multiple symptom domains, including all 10 MADRS items. The strongest treatment effect was found in the Retardation cluster, suggesting that fatigueand cognition-related symptoms of depression may be particularly responsive to levomilnacipran ER.

\section{Disclosures}

Roger McIntyre has the following disclosures: consulting and speaker, consulting fee: AstraZeneca, Eli Lilly, Johnson \& Johnson, Takeda, Pfizer, Lundbeck, Otsuka, Sunovion, Allergan, and BMS. Carl Gommoll has the following disclosure: Actavis pic, employee, salary, stock/stock options. Changzheng Chen has the following disclosure: Actavis pic, employee, salary, stock/stock options. Adam Ruth has the following disclosure: Prescott Medical Communications Group (at time of the studies), a contractor of Forest Research Institute, employee, salary.

\section{REFERENCES:}

1. Rush AJ. The varied clinical presentations of major depressive disorder. J Clin Psychiatry. 2007; 68(Suppl 8): 4-10.

2. Fried EI, Nesse RM. Depression is not a consistent syndrome: an investigation of unique symptom patterns in the $\mathrm{STAR}^{*} \mathrm{D}$ study. J Affect Disord. 2014; 172C: 96-102.

3. Nutt DJ. Relationship of neurotransmitters to the symptoms of major depressive disorder. J Clin Psychiatry. 2008; 69(Suppl E1): 4-7.

4. Suzuki A, Aoshima T, Fukasawa T, et al. A three-factor model of the MADRS in major depressive disorder. Depress Anxiety. 2005; 21(2): 95-97.

5. Paavonen V, Kampman O, Illi A, et al. A cluster model of temperament as an indicator of antidepressant response and symptom severity in major depression. Psychiatry Investig. 2014; 11(1): 18-23.

6. Schacht A, Gorwood P, Boyce P, Schaffer A, Picard H. Depression symptom clusters and their predictive value for treatment outcomes: results from an individual patient data meta-analysis of duloxetine trials. J Psychiatr Res. 2014; 53: 54-61.

7. van Loo HM, Cai T, Gruber MJ, et al. Major depressive disorder subtypes to predict long-term course. Depress Anxiety. 2014; 31(9): 765-777.

8. McIntyre RS, O'Donovan C. The human cost of not achieving full remission in depression. Can J Psychiatry. 2004; 49(3 Suppl 1): 10S-16S.

9. Romera I, Perez V, Ciudad A, et al. Residual symptoms and functioning in depression, does the type of residual symptom matter? A post-hoc analysis. BMC Psychiatry. 2013; 13: 51.

10. Fava M, Ball S, Nelson JC, et al. Clinical relevance of fatigue as a residual symptom in major depressive disorder. Depress Anxiety. 2014; 31(3): 250-257. 
11. Bortolato B, Carvalho AF, McIntyre RS. Cognitive dysfunction in major depressive disorder: a state-of-the-art clinical review. CNS Neurol Disord Drug Targets. 2014; 13(10): 1804-1818.

12. Asnis G, Bose A, Gommoll C, Chen C, Greenberg WM. The efficacy and safety of levomilnacipran SR $40 \mathrm{mg}, 80 \mathrm{mg}$, or $120 \mathrm{mg}$ in major depressive disorder: a phase III, randomized, double-blind, placebo-controlled study. J Clin Psychiatry. 2013; 74(3): 242-248.

13. Bakish D, Bose A, Gommoll C, et al. Levomilnacipran ER $40 \mathrm{mg}$ and $80 \mathrm{mg}$ in patients with major depressive disorder: a phase III, randomized, double-blind, fixed-dose, placebo-controlled study. J Psychiatry Neurosci. 2014; 39(1): 40-49.

14. Sambunaris A, Bose A, Gommoll C, et al. A phase III, double-blind, placebo-controlled, flexible-dose study of levomilnacipran ER in patients with major depressive disorder. J Clin Pscyhopharmacol. 2014; 34(1): 47-56.

15. Montgomery SA, Mansuy L, Ruth A, et al. Efficacy and safety of levomilnacipran sustained release in moderate to severe major depressive disorder: a randomized, double-blind, placebocontrolled, proof-of-concept study. J Clin Psychiatry. 2013; 74(4): 363-369.

16. Montgomery SA, Asberg M. A new depression scale designed to be sensitive to change. Br J Psychiatry. 1979; 134(4): 382-389.

17. Gommoll CP, Greenberg WM, Chen C. A randomized, double-blind, placebo-controlled study of flexible doses of levomilnacipran ER (40-120 mg/day) in patients with major depressive disorder. Journal of Drug Assessment. 2014; 3(1): 10-19.

18. Bech P, Tanghøj P, Andersen HF, Overø K. Citalopram doseresponse revisited using an alternative psychometric approach to evaluate clinical effects of four fixed citalopram doses compared to placebo in patients with major depression. Psychopharmacology (Berl). 2002; 163(1): 20-25.

19. Treadway MT, Zald DH. Reconsidering anhedonia in depression: lessons from translational neuroscience. Neurosci Biobehav Rev. 2011; 35(3): 537-555.

20. Montgomery SA, Gommoll CP, Chen C, Greenberg WM. Efficacy of levomilnacipran extended-release in major depressive disorder: pooled analysis of 5 double-blind, placebo-controlled trials. CNS Spectr. 2015; 20(2): 148-156.

21. Sakurai H, Uchida H, Abe T, et al. Trajectories of individual symptoms in remitters versus non-remitters with depression. J Affect Disord. 2013; 151(2): 506-513.

22. Szegedi A, Jansen WT, van Willigenburg AP, et al. Early improvement in the first 2 weeks as a predictor of treatment outcome in patients with major depressive disorder: a meta-analysis including 6562 patients. J Clin Psychiatry. 2009; 70(3): 344-353.

23. Zimmerman M, Posternak MA, Chelminski I. Derivation of a definition of remission on the Montgomery-Asberg depression rating scale corresponding to the definition of remission on the Hamilton rating scale for depression. J Psychiatr Res. 2004; 38(6): 577-582.

24. Bech P, Tanghøj P, Cialdella P, Andersen HF, Pedersen AG. Escitalopram dose-response revisited: an alternative psychometric approach to evaluate clinical effects of escitalopram compared to citalopram and placebo in patients with major depression. Int J Neuropsychopharmacol. 2004; 7(3): 283-290.

25. Der-Avakian A, Markou A. The neurobiology of anhedonia and other reward-related deficits. Trends Neurosci. 2012; 35(1): 68-77.

26. Higuchi H, Sato K, Yoshida K, et al. Predictors of antidepressant response to fluvoxamine obtained using the three-factor structures of the Montgomery and Asberg Depression Rating Scale for major depressive disorders in Japanese patients. Psychiatry Clin Neurosci. 2008; 62(3): 301-306.

27. Okazaki M, Tominaga K, Higuchi H, et al. Predictors of response to electroconvulsive therapy obtained using the three-factor structure of the Montgomery and Asberg Depression Rating Scale for treatment-resistant depressed patients. JECT. 2010; 26(2): 87-90.

28. Alonzo A, Chan G, Martin D, Mitchell PB, Loo C. Transcranial direct current stimulation (tDCS) for depression: analysis of response using a three-factor structure of the Montgomery-Asberg depression rating scale. J Affect Disord. 2013; 150(1): 91-95.

29. Gold PW, Chrousos GP. Organization of the stress system and its dysregulation in melancholic and atypical depression: high vs low CRH/NE states. Mol Psychiatry. 2002; 7(3): 254-275.

30. Rapaport MH, Thase ME. Translating the evidence on atypical depression into clinical practice. J Clin Psychiatry. 2007; 68(Suppl 3): 31-36.

31. Howland RH, Wilson MG, Kornstein SG, et al. Factors predicting reduced antidepressant response: experience with the SNRI duloxetine in patients with major depression. Ann Clin Psychiatry. 2008; $20(4)$ : 209-218.

32. Sambunaris A, Gommoll C, Chen C, Greenberg WM. Efficacy of levomilnacipran extended-release in improving functional impairment associated with major depressive disorder: pooled analyses of five double-blind, placebo-controlled trials. Int Clin Psychopharmacol. 2014; 29(4): 197-205.

33. Cutler AJ, Gommoll CP, Chen C, Greenberg WM, Ruth A. Levomilnacipran ER treatment in patients with major depressive disorder: improvements in functional impairment categories. Prim Care Companion CNS Disord. 2015; 17(3). doi:10.4088/ PCC.14m01753.

34. Lam RW, Filteau MJ, Milev R. Clinical effectiveness: the importance of psychosocial functioning outcomes. J Affect Disord. 2011; 132(Suppl 1): S9-S13. 